\begin{tabular}{|c|c|c|}
\hline \multirow{3}{*}{$\begin{array}{r}\text { Case Reports in } \\
\text { Gastroenterology }\end{array}$} & \multirow{2}{*}{\multicolumn{2}{|c|}{ Case Rep Gastroenterol 2018;12:76-84 }} \\
\hline & & \\
\hline & $\begin{array}{l}\text { DOI: } 10.1159 / 000481937 \\
\text { Publisned onilne: February } 21,2018\end{array}$ & $\begin{array}{l}\text { (c) } 2018 \text { The Author(s) } \\
\text { Published by S. Karger AG, Basel } \\
\text { www.karger.com/crg }\end{array}$ \\
\hline & \multicolumn{2}{|c|}{$\begin{array}{l}\text { This article is licensed under the Creative Commons Attribution-NonCommercial } 4.0 \\
\text { International License (CC BY-NC) (http://www.karger.com/Services/OpenAccessLicense). } \\
\text { Usage and distribution for commercial purposes requires written permission. }\end{array}$} \\
\hline
\end{tabular}

\title{
Successful Fecal Microbiota Transplantation in a Patient with Severe Complicated Clostridium difficile Infection after Liver Transplantation
}

\author{
Kai Markus Schneider ${ }^{a} \quad$ Theresa H. Wirtz $^{a}$ Daniela Kroy ${ }^{a}$ \\ Stefanie Albers ${ }^{a} \quad$ Ulf Peter Neumann ${ }^{c} \quad$ Till Strowig $^{b} \quad$ Gernot Sellge $^{a}$ \\ Christian Trautwein ${ }^{\mathrm{a}}$ \\ ${ }^{a}$ Department of Medicine III, University Hospital RWTH Aachen, Aachen, Germany; \\ ${ }^{b}$ Helmholtz Centre for Infection Research, Braunschweig, Germany; ${ }^{C}$ Department of \\ Visceral and Transplantation Surgery, University Hospital RWTH Aachen, \\ Aachen, Germany
}

\section{Keywords}

Fecal microbiota transplantation - Clostridium difficile infection · Liver transplantation . Microbiota $\cdot$ Pseudomembranous colitis

\begin{abstract}
Clostridium difficile infection (CDI) represents one of the most common healthcare-associated infections. Due to increasing numbers of recurrences and therapy failures, CDI has become a major disease burden. Studies have shown that fecal microbiota transplantation (FMT) can both be a safe and highly efficacious therapy for patients with therapy-refractory CDI. However, patients undergoing solid organ transplantation are at high risk for CDI due to long-term immunosuppression, previous antibiotic therapy, and proton pump inhibitor use. Additionally, these patients may be especially prone to adverse events related to FMT. Here, we report a successful FMT in a patient with severe therapy-refractory CDI after liver transplantation.

(C) 2018 The Author(s)

Published by S. Karger AG, Basel
\end{abstract}




\section{Introduction}

Over the last decade, there has been an alarming increase in the incidence of pseudomembranous colitis caused by Clostridium difficile infection (CDI), making $C$. difficile (CD) the most common cause of nosocomial diarrhea, associated with increases in mortality and economic costs [1]. Up to $35 \%$ of patients suffer from recurrent infections after initial CDI treatment due to persistence of spores or reinfection [2]. Standard antibiotic treatment not only targets pathogenic $\mathrm{CD}$ but also perpetuates the risk for reinfection by further reducing the diversity of intestinal microbiota [3]. Accordingly, after the first 2 or 3 recurrences, $65 \%$ of the patients face multiple recurrences [4-7].

Solid organ transplantation (SOT) patients have a particularly high risk of CDI, for various reasons: immunosuppressive agents attenuate immune surveillance, enabling bacterial pathogens, such as CD, to evade natural immunity and facilitate bacterial infection. Most notably, the associated frequent need for antibiotic treatment per se represents the highest risk factor for CDI [8]. Not only antibiotics but also proton pump inhibitors significantly raise the likelihood of bacterial infection [9]. Altogether, antibiotic treatment, immunosuppression, and proton pump inhibitors disturb the intricate homeostasis between the host's mucosal immune system and intestinal microbiota promoting overexpansion of $\mathrm{CD}$ and overall disease development. It is, therefore, plausible that restoring the physiological microbiota composition and fine immunological orchestration in the gut by fecal microbiota transplantation (FMT) may be particularly critical for transplant patients.

The first reports of FMT date back as early as 1958 [10]; since then, there have been numerous reports, case series, and the clinical landmark trial by Van Nood et al. [11] demonstrating that FMT is both highly efficacious and safe. Although immunocompromised transplant patients bear a particularly high risk of developing CDI, international guidelines lack clear recommendations regarding FMT in SOT recipients due to the potential risk of adverse events [12]. A recent retrospective analysis has demonstrated the safety and efficacy of FMT in a cohort of immunocompromised patients [13]. However, at present, clinical experience with FMT in solid organ recipients and high-risk settings is still limited. Here, we report a case of successful FMT in a liver transplant patient with severe CDI complicated by acute kidney injury (AKI).

\section{Clinical Case Report}

A 47-year-old Caucasian female received allogeneic liver transplantation at our transplantation center in March 2016 due to decompensated alcoholic liver cirrhosis (Child class C). The early postoperative course was unremarkable for any critical incidents. In May 2016, the patient first presented at our hospital with abdominal cramping and diarrhea up to 7 times per day. At this time, the patient suffered from the diagnosis of CD colitis for the first time, resulting in an antibiotic treatment with oral metronidazole $500 \mathrm{mg}$ t.i.d. for 10 days. A second episode of CDI followed in June 2016, and she was started on oral vancomycin 125 mg q.i.d. Due to therapeutic failure, the treatment regimen was switched to a combination therapy consisting of fidaxomicin and metronidazole, leading to clinical remission. The following clinical course was complicated by acute transplant rejection in September 2016 under chronic immunosuppressive therapy with tacrolimus. Therefore, oral steroids were added and tapered carefully. At this time, the patient additionally developed a nosocomial pneumonia which was treated with tazobactam/piperacillin. 
In October 2016, the patient again presented with severe abdominal distension, ascites, pain, and diarrhea up to 10 times per day. Meanwhile, she had developed life-threatening cachexia and a severely reduced general state of health. Upon admission, her body mass index was 12.5 , and her laboratory tests showed severe leukocytosis $(32.7 / \mathrm{nL})$ and AKI (creatinine $2.67 \mathrm{mg} / \mathrm{dL}$, urea $227 \mathrm{mg} / \mathrm{dL}$ ) (Fig. 1). Due to her history of recurrent and refractory CDI, we suspected a severe course of CDI (see online suppl. Table 2; for all online suppl. material, see www.karger.com/doi/10.1159/000481937), which was characterized by leukocytosis as depicted in Figure 1. Based on ultrasound findings (Fig. 2) of an increased wall thickness ( $5 \mathrm{~mm}$ ) and hyperemia of the wall of the sigmoid colon, the patient was empirically started on oral vancomycin $250 \mathrm{mg}$ q.i.d. A stool sample tested positive for hypertoxinproducing CD species. As symptoms did not resolve after vancomycin treatment, we evaluated her for FMT. After discussing potential risks and benefits of FMT and informed consent to FMT, the patient's brother was chosen as donor and a disease screen was performed according to our FMT standard operating procedures.

Flexible sigmoidoscopy on day 5 of admission showed severe colitis with confluent pseudomembranes covering the entire mucosa, friability of the mucosa with bleeding upon contact, and extensive edema (Fig. 3). In line with this, the microscopic architecture of the colonic mucosa was disrupted and displayed severe inflammatory cell infiltration and complete loss of colonic crypts as well as goblet cells (Fig. 3). A stool sample was obtained during sigmoidoscopy to analyze the microbiota composition using 16s rRNA gene sequencing. We first analyzed alpha diversity (as measured by observed species, Shannon index), which revealed extremely low complexity of microbiota (Fig. 4). The microbiota community was characterized by untypically high abundances of the families Alcaligenaceae, Enterobacteriaceae, Enterococcaceae, and Veillonellaceae, which are all associated with dysbiosis in the intestine and contain members with pathogenic potential (Fig. 4). Due to the risk of perforation, we abstained from performing a higher sigmoidoscopy and decided to perform FMT in the higher rectum. Twenty-four hours after FMT, the patient reported a marked improvement of clinical symptoms with reduced abdominal cramps and decreased stool frequency (Fig. 1). This clinical improvement was reflected in reduced white blood cell count and improved kidney function (Fig. 1).

Due to the high degree of mucosal injury, the encouraging treatment response, and a slight increase in stool frequency on day 10, we decided to perform a second FMT via oral capsules 6 days after endoscopic intervention. At day 16, the patient showed an almost complete remission correlating with normalized white blood cell count and blood urea (Fig. 1). A control colonoscopy showed a healthy mucosa with only very few residual pseudomembranes (Fig. 3). At this time, we could observe a marked increase in microbial diversity; however, alpha diversity did not reach the levels of the donor community (Fig. 4). We detected increased abundances of bacteria associated with a healthy microbiome (orders Bacteroidales and Clostridiales), remaining elevated levels of Enterobacteriaceae and Enterococcaceae, but disappearance of Veillonellaceae (Fig. 4). The patient did not complain about further diarrhea and was discharged from our hospital in a substantially improved general state of health. In an outpatient follow-up 8 weeks after FMT, body weight had already increased by $25 \%$ (Fig. 5). 


\section{Discussion and Implications}

Our case report shows that FMT can represent both a safe and efficacious therapy for severe complicated CDI even in an acutely ill patient who underwent SOT. During the last decade, the introduction of FMT into clinical practice through systematic evaluation in randomized trials opened up a new therapeutic avenue for patients suffering from refractory CDI. Widespread use of broad-spectrum antibiotics decreases microbiome species diversity and, thus, renders the patients' intestinal microbiota susceptible to overgrowth with pathogenic CD [14, 15]. Upon admission, our patient presented markedly reduced microbiota diversity with high abundance of potentially pathogenic bacteria. Although our standard antibiotic regimens have proven to be highly effective in most cases of CDI, there is an alarming increase in infections refractory to standard therapy [16]. In part, this can be attributed to new highly virulent strains of CD. On the other hand, recurrent antibiotic treatment and immunosuppression after liver transplantation promote intestinal dysbiosis, which increases the risk for CDI [14]. Our patient already underwent multiple courses of antibiotic treatment and presented in a highly compromised general state of health infected with a highly virulent strain of CD.

Today, a huge body of evidence underscores the safety and efficacy of restoring intestinal microbiota composition by FMT. SOT patients per se are more susceptible to CDI due to recurrent phases of hospitalization and use of immunosuppressive agents. While the general prevalence of CDI among hospitalized patients is reported to be $0.9 \%$ [17], overall solid organ recipients' risk for CDI is 7.4\%; in case of liver transplant, it is even higher (9.1\%) [18]. Moreover, CDI is independently associated with higher mortality (adjusted odds ratio 2.48) and complications of the transplanted organ (adjusted odds ratio 1.36), identifying CDI as a feared complication after SOT [19]. Beside the commonly used immunosuppressive agents, especially the initiation of steroids, as described in our case, represents a serious risk factor for CDI as it triples the risk for infection [20]. Combined immunosuppression after SOT both dampens the adaptive and innate immune response, leading to mitigated neutrophil recruitment upon infection and reduced assembly of humeral IgG antibodies against toxin A and B, which has been linked to CDI recurrences [21,22].

As our patient suffered from recurrent CDI, we first decided to max out the different regimens of antibiotic treatment for recurrent severe CDI, including vancomycin and fidaxomicin. The latter represents an alternative for the treatment of recurrent CDI, showing comparable cure rates to vancomycin with significantly fewer patients experiencing recurrence $[23,24]$. However, only 4 weeks after treatment with fidaxomicin, the patient was rehospitalized with another course of CDI worsened by AKI and beginning symptoms of sepsis, which could, therefore, be classified as a complicated CDI. As antibiotics themselves may increase the risk of recurrence by reducing microbiota species diversity and causing intestinal dysbiosis, European guidelines strongly recommend FMT for treating multiple recurrences of CDI [25].

However, next to the desired therapeutic effects of FMT, we had to face the risk of potential adverse events, which warrant careful consideration. This was especially valid for the high-risk setting we were confronted with here: severe therapy-refractory CDI in a SOT patient, which was complicated by AKI. Infusing microbiota during colonoscopy can prompt life-threatening complications, such as systemic infection due to bacterial translocation [26]. In line, there are recommendations against FMT in such settings, and clear guidelines are still missing [27]. A recent retrospective study analyzed the role of FMT for the treatment of CDI in immunocompromised patients. Data from 99 patients were included, showing a cure 
rate of 78\% after single FMT and of $89 \%$ after repeated FMT [28]. In summary, the authors could not detect any adverse events that were related to FMT [13].

However, beside immunosuppression, our patient was also in a severely compromised general state of health and already developed second organ failure. Today, there are still limited data about potential risks of FMT in acutely ill patients with significant comorbidities as those patients have been excluded from initial trials $[2,11]$. It is clear that the procedure is not without risk and the indication should be evaluated carefully. As our patient suffered from an exceptionally severe form of CDI, we decided to abstain from performing a higher sigmoidoscopy and applied microbiota transfer in the rectum. Our patient recovered from clinical symptoms already after the first FMT. As especially acutely ill patients might not show sustained improvement of clinical symptoms after a first FMT [29], we decided to ensure long-term recovery of the patient by performing a second FMT. A therapeutic approach with sequential FMT and combination with antibiotics for severe forms of CDI is described in different case reports as well as a retrospective study [29, 30]. After sequential FMT, our patient showed long-term remission of CDI up to several months until now. The remarkably fast remission of clinical symptoms, decrease of leukocytosis, and recovery from AKI was followed by a body weight gain of $25 \%$ in the next 8 months, leading to marked improvements of general agility and mental health. Our case suggests that a protocol consisting of a first FMT followed by a consolidation therapy with FMT via oral capsule might be an efficacious FMT protocol for patients with severe therapy-refractory CDI.

In conclusion, our case exemplifies that even in a high-risk situation of acute illness FMT can be both a highly efficacious and safe treatment for severe complicated CDI. Further randomized controlled trials or matched case-control series would help to systematically evaluate FMT protocols in this vulnerable subgroup of patients that vitally requires an effective treatment for life-threatening CDI.

\section{Statement of Ethics}

The patient gave consent and encouraged the publication of the presented data.

\section{Disclosure Statement}

All authors declare that they have nothing to disclose.

\section{References}

-1 Surawicz CM, Brandt LJ, Binion DG, Ananthakrishnan AN, Curry SR, Gilligan PH, et al: Guidelines for diagnosis, treatment, and prevention of Clostridium difficile infections. Am J Gastroenterol 2013;108:478-498; quiz 499.

-2 Kelly CP, LaMont JT: Clostridium difficile - more difficult than ever. N Engl J Med 2008;359:1932-1940.

-3 Becattini S, Taur Y, Pamer EG: Antibiotic-induced changes in the intestinal microbiota and disease. Trends Mol Med 2016;22:458-478.

4 McFarland LV, Elmer GW, Surawicz CM: Breaking the cycle: treatment strategies for 163 cases of recurrent Clostridium difficile disease. Am J Gastroenterol 2002;97:1769-1775.

5 Kelly CP: Can we identify patients at high risk of recurrent Clostridium difficile infection? Clin Microbiol Infect 2012;18(suppl 6):21-27.

-6 Bauer MP, Notermans DW, Van Benthem BH, Brazier JS, Wilcox MH, Rupnik M, et al: Clostridium difficile infection in Europe: a hospital-based survey. Lancet 2011;377:63-73. 


\section{Case Reports in \\ Gastroenterology}

Case Rep Gastroenterol 2018;12:76-84

DOI: $10.1159 / 000481937$

(C) 2018 The Author(s). Published by S. Karger AG, Basel www.karger.com/crg

Schneider et al: Successful Fecal Microbiota Transplantation in a Patient with Severe Complicated Clostridium difficile Infection after Liver Transplantation

7 Gough E, Shaikh H, Manges AR: Systematic review of intestinal microbiota transplantation (fecal bacteriotherapy) for recurrent Clostridium difficile infection. Clin Infect Dis 2011;53:994-1002.

-8 Garey KW, Sethi S, Yadav Y, DuPont HL: Meta-analysis to assess risk factors for recurrent Clostridium difficile infection. J Hosp Infect 2008;70:298-304.

-9 Buendgens L, Bruensing J, Matthes M, Dückers H, Luedde T, Trautwein C, et al: Administration of proton pump inhibitors in critically ill medical patients is associated with increased risk of developing Clostridium difficile-associated diarrhea. J Crit Care 2014;29:696.e11-e15.

-10 Eiseman B, Silen W, Bascom G, Kauvar A: Fecal enema as an adjunct in the treatment of pseudomembranous enterocolitis. Surgery 1958;44:854-859.

11 van Nood E, Vrieze A, Nieuwdorp M, Fuentes S, Zoetendal EG, de Vos WM, et al: Duodenal infusion of donor feces for recurrent Clostridium difficile. N Engl J Med 2013;368:407-415.

12 Friedman-Moraco RJ, Mehta AK, Lyon GM, Kraft CS: Fecal microbiota transplantation for refractory Clostridium difficile colitis in solid organ transplant recipients. Am J Transplant 2014;14:477-480.

13 Kelly CR, Ihunnah C, Fischer M, Khoruts A, Surawicz C, Afzali A, et al: Fecal microbiota transplant for treatment of Clostridium difficile infection in immunocompromised patients. Am J Gastroenterol 2014;109:1065-1071.

14 Cammarota G, Ianiro G, Gasbarrini A: Fecal microbiota transplantation for the treatment of Clostridium difficile infection: a systematic review. J Clin Gastroenterol 2014;48:693-702.

15 Youngster I, Mahabamunuge J, Systrom HK, Sauk J, Khalili H, Levin J, et al: Oral, frozen fecal microbiota transplant (FMT) capsules for recurrent Clostridium difficile infection. BMC Med 2016;14:134.

16 Kelly CR, Ihunnah C, Fischer M, Khoruts A, Surawicz C, Afzali A, et al: Fecal microbiota transplant for treatment of Clostridium difficile infection in immunocompromised patients. Am J Gastroenterol 2014;109:1065-1071.

17 Lucado J, Gould C, Elixhauser A: Clostridium difficile infections (CDI) in hospital stays, 2009: statistical brief \#124; in Healthcare Cost and Utilization Project (HUCP) Statistical Briefs. Rockville, MD, Agency for Healthcare Research and Quality (US), 2006-2012. http://www.ncbi.nlm.nih.gov/pubmed/22574332.

-18 Paudel S, Zacharioudakis IM, Zervou FN, Ziakas PD, Mylonakis E: Prevalence of Clostridium difficile infection among solid organ transplant recipients: a meta-analysis of published studies. PLoS One 2015;10:e0124483.

19 Pant C, Anderson MP, O'Connor JA, Marshall CM, Deshpande A, Sferra TJ: Association of Clostridium difficile infection with outcomes of hospitalized solid organ transplant recipients: results from the 2009 Nationwide Inpatient Sample database. Transpl Infect Dis 2012;14:540-547.

-20 Schneeweiss S, Korzenik J, Solomon DH, Canning C, Lee J, Bressler B: Infliximab and other immunomodulating drugs in patients with inflammatory bowel disease and the risk of serious bacterial infections. Aliment Pharmacol Ther 2009;30:253-264.

-21 Kyne L, Warny M, Qamar A, Kelly CP: Association between antibody response to toxin A and protection against recurrent Clostridium difficile diarrhoea. Lancet 2001;357:189-193.

22 Leav BA, Blair B, Leney M, Knauber M, Reilly C, Lowy I, et al: Serum anti-toxin B antibody correlates with protection from recurrent Clostridium difficile infection (CDI). Vaccine 2010;28:965-969.

23 Louie TJ, Miller MA, Mullane KM, Weiss K, Lentnek A, Golan Y, et al: Fidaxomicin versus vancomycin for Clostridium difficile Infection. N Engl J Med 2011;364:422-431.

24 Cornely OA, Crook DW, Esposito R, Poirier A, Somero MS, Weiss K, et al: Fidaxomicin versus vancomycin for infection with Clostridium difficile in Europe, Canada, and the USA: a double-blind, non-inferiority, randomised controlled trial. Lancet Infect Dis 2012;12:281-289.

25 Debast SB, Bauer MP, Kuijper EJ; European Society of Clinical Microbiology and Infectious Diseases: European Society of Clinical Microbiology and Infectious Diseases: update of the treatment guidance document for Clostridium difficile infection. Clin Microbiol Infect 2014;20(suppl 2):1-26.

-26 Wang S, Xu M, Wang W, Cao X, Piao M, Khan S, et al: Systematic review: adverse events of fecal microbiota transplantation. PLoS One 2016;11:e0161174.

-27 Dubberke ER, Burdette SD; AST Infectious Diseases Community of Practice: Clostridium difficile infections in solid organ transplantation. Am J Transplant 2013;13(suppl 4):42-49.

-28 Webb BJ, Brunner A, Ford CD, Gazdik MA, Petersen FB, Hoda D: Fecal microbiota transplantation for recurrent Clostridium difficile infection in hematopoietic stem cell transplant recipients. Transpl Infect Dis 2016;18:628-633.

29 Fischer M, Sipe BW, Rogers NA, Cook GK, Robb BW, Vuppalanchi R, et al: Faecal microbiota transplantation plus selected use of vancomycin for severe-complicated Clostridium difficile infection: description of a protocol with high success rate. Aliment Pharmacol Ther 2015;42:470-476.

-30 Weingarden AR, Hamilton MJ, Sadowsky MJ, Khoruts A: Resolution of severe Clostridium difficile infection following sequential fecal microbiota transplantation. J Clin Gastroenterol 2013;47:735-737. 
Schneider et al.: Successful Fecal Microbiota Transplantation in a Patient with Severe Complicated Clostridium difficile Infection after Liver Transplantation

K.M.S. and T.H.W. share 1st authorship.

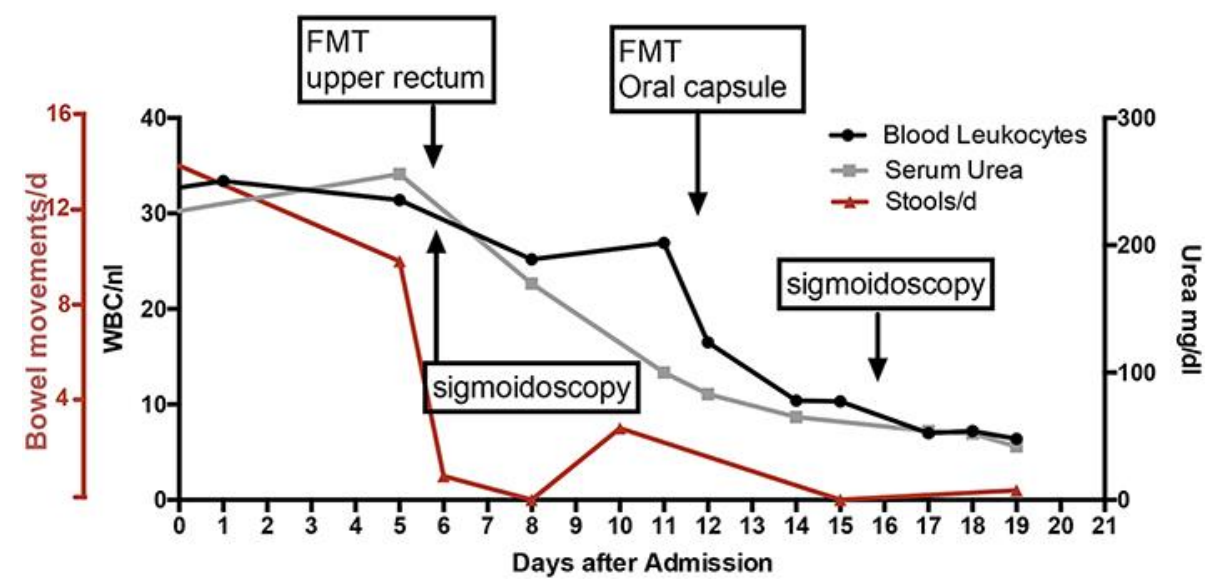

Fig. 1. Course of treatment including bowel movements per day (red line), white blood cell count (WBC/nL, black line), and serum urea level (grey line) from the first day of admission until 1 week after the second FMT. Time points of first and second FMT as well as diagnostic sigmoidoscopy are highlighted. FMT, fecal microbiota transplantation; $d$, day.

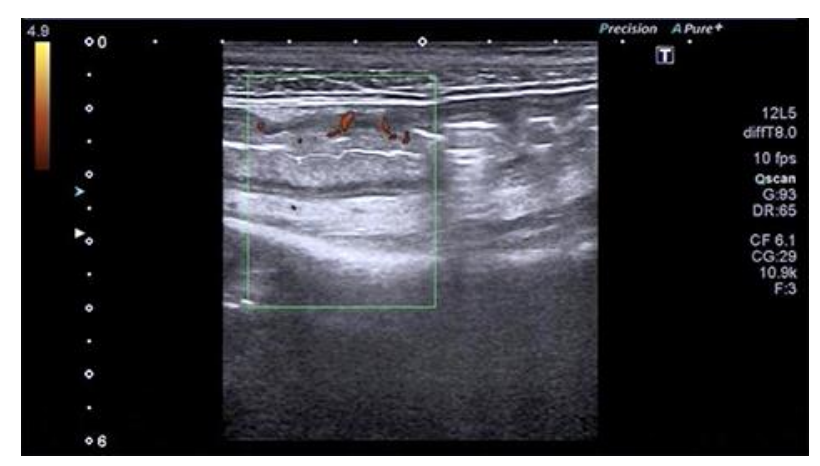

Fig. 2. Abdominal ultrasound images displayed colonic wall thickening as well as hyperemia prior to the first fecal microbiota transplantation. 
Schneider et al.: Successful Fecal Microbiota Transplantation in a Patient with Severe Complicated Clostridium difficile Infection after Liver Transplantation

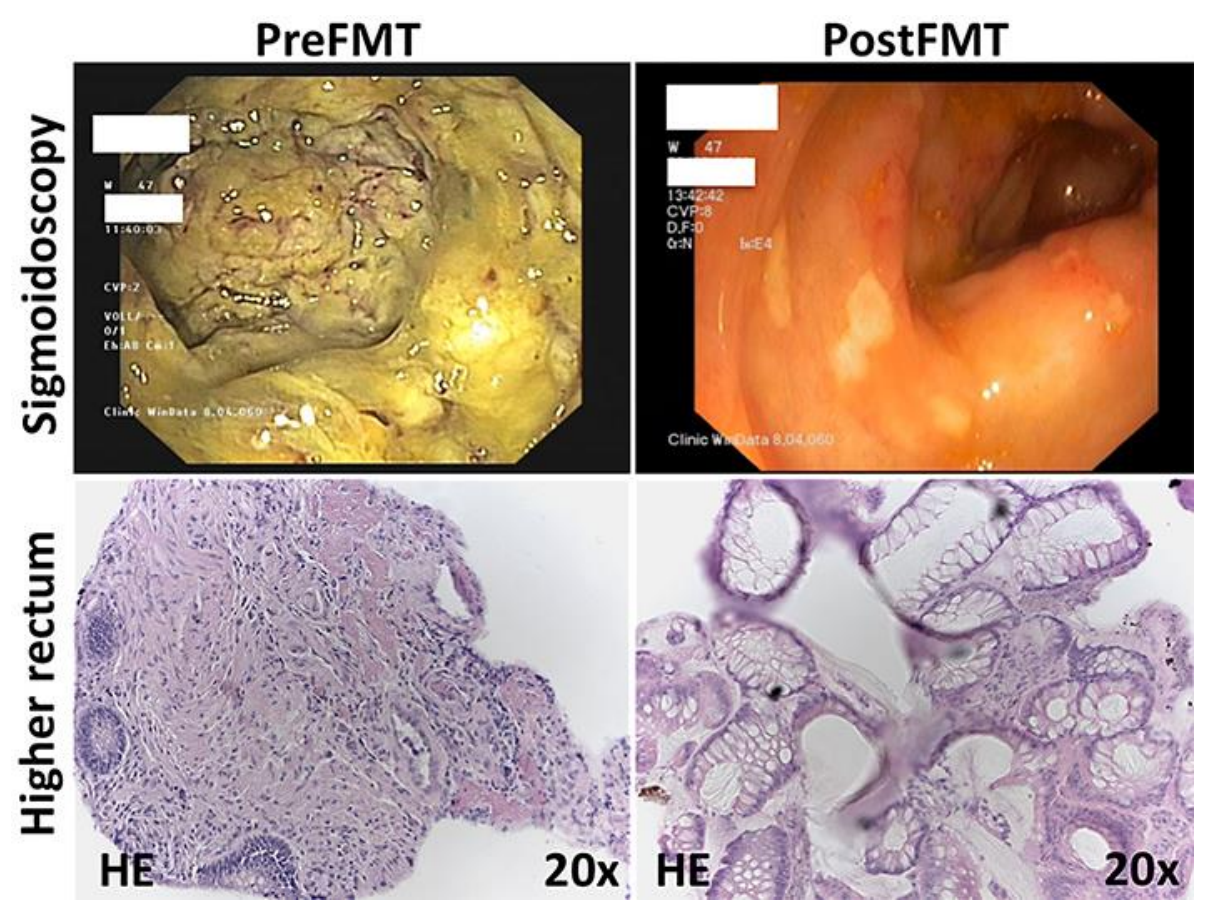

Fig. 3. Representative macroscopic (top) and histologic (bottom) changes of the colonic mucosa pre- and post-FMT. Macroscopic pictures were taken during sigmoidoscopy presenting pseudomembranes as well as contact bleeding in this extensively inflamed mucosa at day 5 of admission before FMT (top left) and only few residual pseudomembranes 4 days after second FMT (top right). HE-stained tissue sections of biopsies taken in the higher rectum during endoscopy at the same time points show relevant infiltration of immune cells and disruption of the mucosal epithelial barrier before FMT (bottom left) as well as recovery of the colonic mucosa after second FMT (bottom right). FMT, fecal microbiota transplantation.
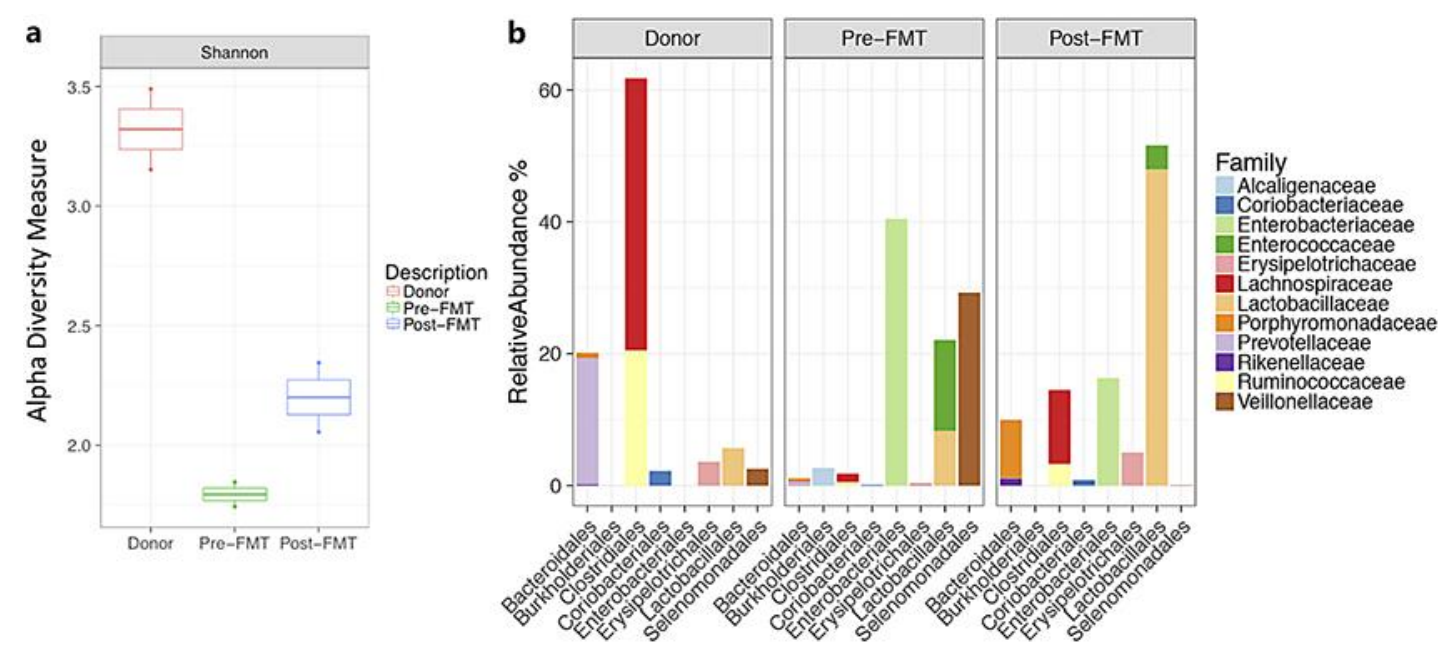

Fig. 4. Analysis of the donor's and patient's microbiota composition pre- and post-FMT. a The overall alpha diversity (Shannon index) of the donor's as well as the patient's microbiota pre- and post-FMT. A marked increase in microbiota diversity can be observed post-FMT. b Gut microbiota composition showing relative abundances in \% on phylogenetic family level. FMT, fecal microbiota transplantation. 
Schneider et al:: Successful Fecal Microbiota Transplantation in a Patient with Severe Complicated Clostridium difficile Infection after Liver Transplantation

\section{BMI Development}

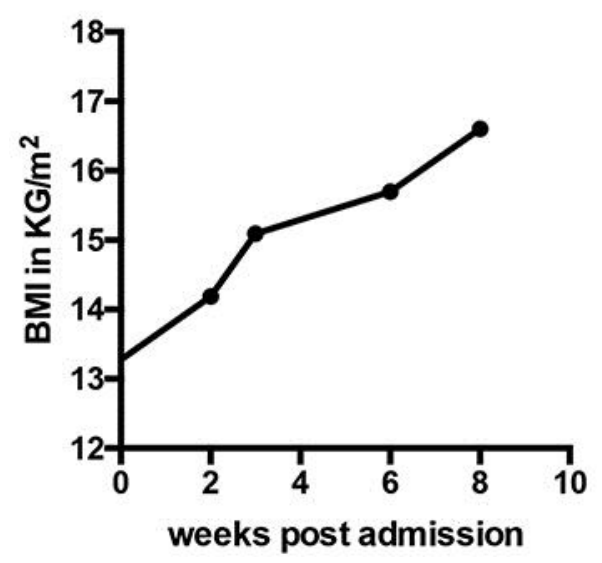

Fig. 5. Increase in patient's body mass index (BMI) in $\mathrm{kg} / \mathrm{m}^{2}$ during 8 weeks of follow-up after initial admission. 